\title{
Diffusion tensor tractography for the surgical management of peripheral nerve sheath tumors
}

\author{
Manfred Schmidt, MD, ${ }^{1}$ Gregor Kasprian, MD, ${ }^{2}$ Gabriele Amann, MD, ${ }^{3}$ Dominik Duscher, MD, ${ }^{1}$ and \\ Oskar C. Aszmann, MD4
}

${ }^{1}$ Section of Plastic and Reconstructive Surgery, General Hospital Linz, Austria; and Departments of ${ }^{2}$ Radiology and ${ }^{3} \mathrm{Clinical}$ Pathology and ${ }^{4}$ Division of Plastic and Reconstructive Surgery, Medical University of Vienna, Austria

\begin{abstract}
OBJECT Peripheral nerve sheath tumors (PNSTs) are uncommon but bear a significant risk of malignancy. High-resolution MRI is the standard technique for characterizing PNSTs. However, planning the appropriate extent of resection and subsequent reconstructive strategies is highly dependent on the intraoperative findings because preoperative MRI evaluation can be insufficient. Diffusion tensor tractography (DTT) represents a recently developed advanced MRI technique that reveals the microstructure of tissues based on monitoring the random movement of water molecules. DTT has the potential to provide diagnostic insights beyond conventional MRI techniques due to its mapping of specific fibrillar nerve structures. Here, DTT was applied to evaluate PNSTs and to examine the usefulness of this method for the correct delineation of tumor and healthy nerve tissue and the value of this information in the preoperative planning of surgical interventions.
\end{abstract}

METHODS In this prospective study, patients with the clinical symptoms of a PNST were investigated using DTT 3-Tesla MRI scans. Image data processing and tractography were performed using the FACT (fiber assessment by continuous tracking) algorithm and multiple-regions-of-interest approach. The surgical findings were then compared with the results of the DTT MRI scans. Preoperative fascicle visualization and the correlation with the intraoperative findings were graded.

RESULTS In a 21-month period, 12 patients with PNSTs were investigated (7 female and 5 male patients with a mean age of $46.2 \pm 19.2$ years). All patients underwent surgical removal of the tumor. Schwannoma was the most common benign histopathological finding ( $n=7)$, whereas 2 malignant lesions were detected. In 10 of 12 patients, good preoperative nerve fascicle visualization was achieved using DTT scans. In 9 of 10 patients with good preoperative fascicle visualization, good intraoperative correlation between the DTT scans and surgical anatomy was found.

CONCLUSIONS DTT properly visualizes the peripheral nerve fascicles and their correct anatomical relation to PNST. DTT represents a promising new method for the preinterventional planning of nerve tumor resection.

http://thejns.org/doi/abs/10.3171/2015.6.FOCUS15228

KEY WORDS peripheral nerve sheath tumor; diffusion tensor tractography; magnetic resonance imaging; schwannoma; neurofibroma

$\mathrm{P}$ ERIPHERAL nerve sheath tumors (PNSTs) are uncommon. ${ }^{7,10}$ They comprise less than $5 \%$ of tumors of the hand and upper extremities but bear a significant risk of malignancy with a benign-to-malignant ratio of 9:1.,11,14 The risk of developing a malignant PNST is estimated at $0.001 \%$ in the general population, although the incidence is up to $8 \%$ to $13 \%$ in patients with neurofibromatosis Type $1 . ?$

Although clinical examination, ultrasound investigation, and electrodiagnostic studies are the first steps for diagnosing PNSTs, high-resolution MRI is needed to characterize the tumor mass and extent of fascicular involve- ment. ${ }^{7,8,13,14}$ However, the low specificity of MRI scans for the correct discrimination of benign and malignant PNST has been reported. ${ }^{7,8,14}$ Furthermore, there is often great difficulty in correctly delineating the tumor and healthy nerve structures. In particular, resection of a benign PNST and the preservation of unaffected nerve fascicles are of the utmost importance to maintaining neuromuscular function..$^{5,11,16}$ With current standard diagnostics, planning the appropriate extent of resection and subsequent reconstructive strategies is still highly dependent on the intraoperative findings because preoperative MRI evaluation can be insufficient (Fig. 1). ${ }^{3}$

ABBREVIATIONS DTI = diffusion tensor imaging; DTT = diffusion tensor tractography; PNST = peripheral nerve sheath tumor.

SUBMITTED May 1, 2015. ACCEPTED June 17, 2015.

INCLUDE WHEN CITING DOI: 10.3171/2015.6.FOCUS15228. 
Diffusion tensor tractography (DTT) represents a recently developed noninvasive MRI technique that reveals the microstructure of tissues based on monitoring the random movement of water molecules. ${ }^{9}$ DTT has the potential to achieve diagnostic insights beyond conventional (anatomical) MRI techniques due to its functional mapping of specific fibrillar nerve structures. ${ }^{21}$ Clinically, this technique is mainly used for imaging the central nervous system and soft-tissue lesions..$^{2,6,15,20,23}$ Experimentally, it has been successfully applied to visualize degeneration and regeneration in peripheral nerves by tracking nerve fibers. ${ }^{22,19}$ However, only sparse evidence exists regarding the value of DTT for the assessment of peripheral nerves and their malignancies.

Here, we applied DTT for the evaluation of PNSTs and tumorlike conditions. Our purpose was to prospectively examine the usefulness of DTT for the correct delineation of tumors and healthy nerve tissue and the value of this information in the preoperative planning of surgical interventions.

\section{Methods}

In this prospective study, patients with clinical symptoms of a PNST were investigated using DTT MRI scans. Written informed consent was obtained from every patient, and the study was approved by the institutional review committee of the Medical University of Vienna.

Patients were imaged using 3-Tesla MRI and an axial echo-planar single-shot diffusion tensor sequence $(\mathrm{b}$ $=0 / 700 ; \mathrm{FOV}=160 \times 160 \times 112 \mathrm{~mm}$; matrix $64 \times 64$; $16 / 32$ diffusion-encoding directions) coregistered with conventional MR sequences, which is used as our standardized institutional soft-tissue tumor MRI protocol. Image data postprocessing and tractography of the major peripheral nerves ( $>3 \mathrm{~mm}$ in diameter) was performed using the FACT (fiber assessment by continuous tracking) algorithm and multiple-regions-of-interest approach. The nerve trajectories were 3D visualized using Philips Achieva software (release 2.1.1.0). If the clinical diagnosis could be confirmed, the patient was scheduled for surgery according to the study protocol. Three-dimensional video sequences visualizing the relevant surgical anatomy in proximity to the tumor were produced and studied by the surgeon preoperatively (Video 1).

VIDEO 1. Video sequence available for the preoperative planning of tumor removal in Case 2. The 3D simulation allows ideal visualization of the relevant anatomical structures and accurate planning of the surgical approach. Copyright Oskar C. Aszmann. Used with permission. Click here to view with Media Player. Click here to view with Quicktime.

Intraoperatively, the course and position of intact nerve fascicles in relation to the tumor were precisely documented by taking representative photographs. After removal, the tumor was marked with sutures to allow correct preparation by the pathologist. The surgical findings were then compared with the results of the DTT MRI scans by 2 independent investigators. Preoperative fascicle visualization and the correlation with the intraoperative findings was graded as good, poor, or missing (Table 1).
TABLE 1. Definition of correlation degree between DTT scans and operative findings

\begin{tabular}{cl}
\hline Correlation Degree & \multicolumn{1}{c}{ Definition } \\
\hline Good & Fascicle course and position match correctly \\
\hline Poor & $\begin{array}{c}\text { Not every fascicle course \& position match } \\
\text { correctly }\end{array}$ \\
\hline None & No match \\
\hline
\end{tabular}

\section{Results}

In a 21-month period, 14 patients with PNST were investigated using DTT MRI scans. In 2 patients, no tumor removal was performed and they were consequently excluded from the study. There were 7 female and 5 male patients, and the mean age at surgery was $46.2 \pm 19.2$ years (range 16-68 years; see Table 2 for a synopsis of the patient data). The peroneal nerve was most commonly affected $(n=4)$, whereas all other PNSTs occurred in different nerves (Table 2). Twelve patients underwent subsequent surgical removal of the tumor. Schwannoma was the most common benign histopathological finding (7 of 12 tumors), whereas 2 malignant PNSTs were detected (see Table 2 for the detailed histological results).

In 10 of 12 patients, good preoperative nerve fascicle visualization could be achieved using DTT scans. In 2 patients, only poor or no preoperative fascicle visualization was possible. In 9 of 10 patients with good preoperative fascicle visualization, a good intraoperative correlation between DTT scans and surgical anatomy was found (Table 3).

\section{Discussion}

PNSTs are rare and mostly benign. ${ }^{7,11,17}$ However, their removal may result in considerable loss of function. ${ }^{5,11,16}$ Therefore, preoperative nerve visualization and accurate planning of surgery and reconstruction are of utmost importance. Up-to-date ultrasound and MRI scans have been used for preoperative imaging despite a considerable lack of adequate differentiation of the tumor mass and healthy nerve fibers. $7,8,13,14$

Recently, advanced MRI technology has been introduced that allows the utilization of DTT for tracing specific neural pathways, e.g., the corticospinal tract in the brain or spinal cord. ${ }^{15,20,23}$ This approach is based on diffusion tensor imaging (DTI), a noninvasive technique that reveals the microstructure of tissues based on monitoring the movement of water molecules. The procedure relies on the detection of thermally driven random motion (diffusion) of water molecules in the direction of the field gradient. Distinct tissues (e.g., nerve tissue) exhibit specific structural properties that ease diffusion in 1 direction while limiting it in another, which is called "anisotropy." Because the peripheral nerves are composed of fascicles containing nerve fibers, water molecules tend to move in the direction of the axons, which is called "anisotropic diffusion." These tissue characteristics allow DTI to contrast and image tissues with different anisotropic features (e.g., nerve tracts and surrounding tissue). ${ }^{12,18,19}$ 
TABLE 2. Synopsis of patients with PNSTs

\begin{tabular}{ccllll}
\hline Case No. & Age $(\mathrm{yrs})$ & Sex & \multicolumn{1}{c}{ Nerve } & Side & Tumor Histology \\
\hline 1 & 42 & F & C-7 root & Right & Plexiform NF \\
\hline 2 & 60 & F & Median & Left & Ancient schwannoma \\
\hline 3 & 40 & F & Frontal & Left & NF \\
\hline 4 & 16 & M & Peroneal & Left & MPNST \\
\hline 5 & 58 & M & Musculocutaneus & Right & Perineurioma \\
\hline 6 & 17 & M & Cranial nerve X & Right & Focal MPNST \\
\hline 7 & 68 & F & Tibial & Left & Schwannoma \\
\hline 8 & 58 & M & Peroneal & Left & Ancient schwannoma \\
\hline 9 & 57 & M & Femoral & Right & Schwannoma \\
\hline 10 & 21 & F & Radial & Left & Schwannoma \\
\hline 11 & 47 & F & Peroneal & Right & Schwannoma \\
\hline 12 & 68 & F & Peroneal & Left & Ancient schwannoma \\
\hline
\end{tabular}

MPNST = malignant PNST; NF = neurofibroma.

DTT is routinely used in neurosurgery to delineate functionally important white matter tracts during surgical planning; however, for the examination of the peripheral nerves, this technology is still used mainly in an experimental fashion. ${ }^{1,12,19}$ Only recent evidence begins to indicate that DTT might have a role in the diagnosis of PNSTs. ${ }^{3,4}$ Here, we applied DTT to PNSTs in order prospectively examine the usefulness of this technology for the correct delineation of tumorous and healthy nerve tissues and the value of this information for preoperative planning.

DTT was found to be capable of properly visualizing intact nerve fascicles and correctly delineating healthy nerve tissue and tumors across the vast majority of PNST patients. In 10 of 12 patients, DTT scans with good preoperative nerve fascicle visualization were obtained. In 9 of 10 patients with good preoperative fascicle visualization, a good intraoperative correlation between the DTT scans and surgical anatomy could be found (Figs. 2-4). One patient showed extensive diffuse nerve fiber splitting around

TABLE 3. DTT nerve visualization and clinical correlation

\begin{tabular}{ccc}
\hline Case No. & $\begin{array}{c}\text { Preoperative Nerve } \\
\text { Visualization }\end{array}$ & $\begin{array}{c}\text { Correlation w/ Intraop } \\
\text { Finding }\end{array}$ \\
\hline 1 & Good & Good \\
\hline 2 & Good & Good \\
\hline 3 & No & Good \\
\hline 4 & Good & Poor \\
\hline 5 & Good & Good \\
\hline 6 & No & Good \\
\hline 7 & Good & Good \\
\hline 8 & Good & Good \\
\hline 10 & Good & Good \\
\hline 11 & Good & Good \\
\hline 12 & Good &
\end{tabular}

the tumor, which resulted in the only poor correlation between DTT and surgical findings.

The limitations of DTT were encountered in 3 patients in whom no or only poor fascicle visualization could be archived preoperatively. One patient presented with extensive pelvic neurofibromatosis, which precluded tracking of the pelvic plexus more peripherally than the nerve root level. However, the patient did not undergo subsequent surgery and was therefore excluded from the study. In a patient with 2 neurofibromata smaller than $5 \mathrm{~mm}$ on the forehead and another patient with a tumor adjacent to the big cervical vessels, no fascicle visualization was possible due to the tumor size and location, respectively (Fig. 5).

Electrophysiological mapping of the tumor capsule represents an especially helpful tool for the intraoperative identification of nerve fascicles. ${ }^{22}$ In particular, this technique may be additionally employed if identification

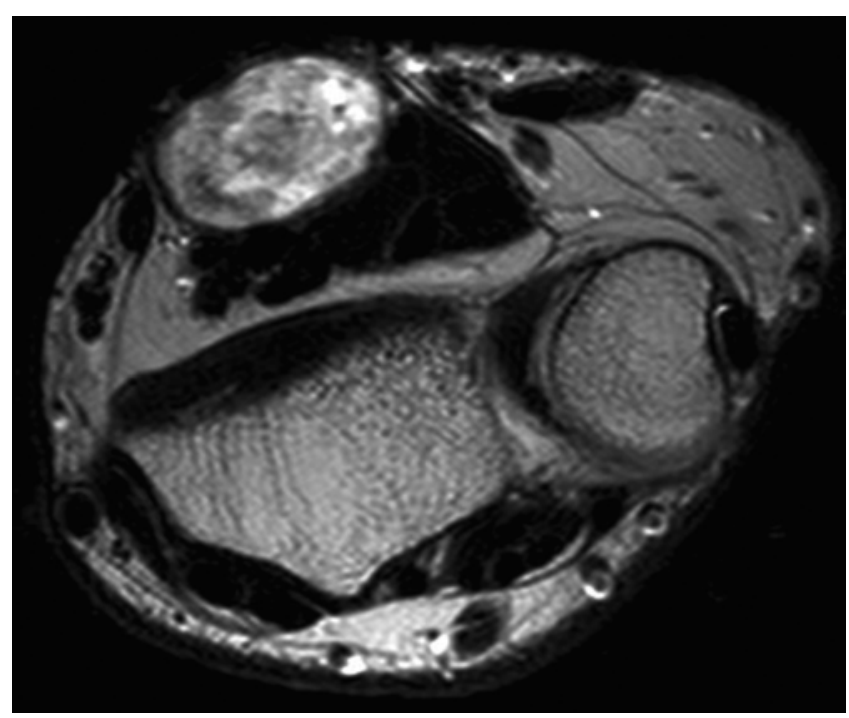

FIG. 1. Case 2. Conventional MRI scan of a schwannoma of the left median nerve at the patient's wrist. The fascicles of the median nerve are barely visible. 

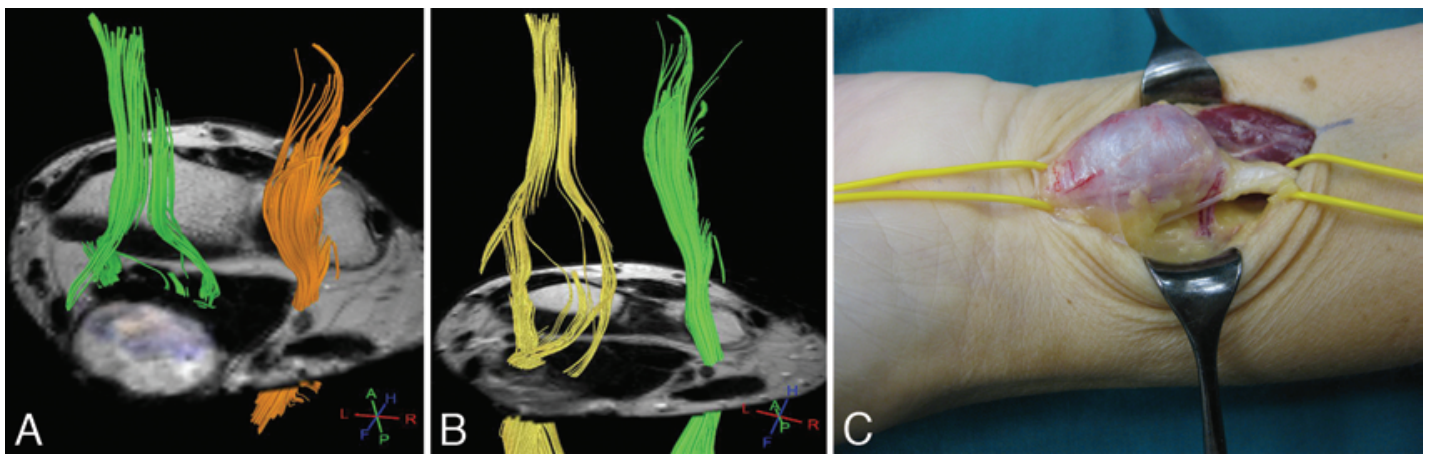

FIG. 2. Case 2. A: Three-dimensional DTT scan of the schwannoma presented in Fig. 1. The median (green) and ulnar nerves (orange) are visualized. The main trunk of the median nerve is split by the tumor into 2 fascicle groups that pass the tumor mass dorsally and radiolaterally, respectively. B: Three-dimensional DTT scan of tumor presented in Figs. 1 and 2A. The fascicles of the median nerve are separated by the tumor and rejoin each other at the level of the wrist joint. The tumor mass is left out by the nerve fibers, and its location can thereby be estimated. C: Intraoperative photograph of the tumor of the left median nerve at the wrist presented in Figs. 1 and 2B. The radiolateral fascicle group and the second fascicle group, which passes the tumor mass dorsally, can be seen (the left side of the picture is distal).
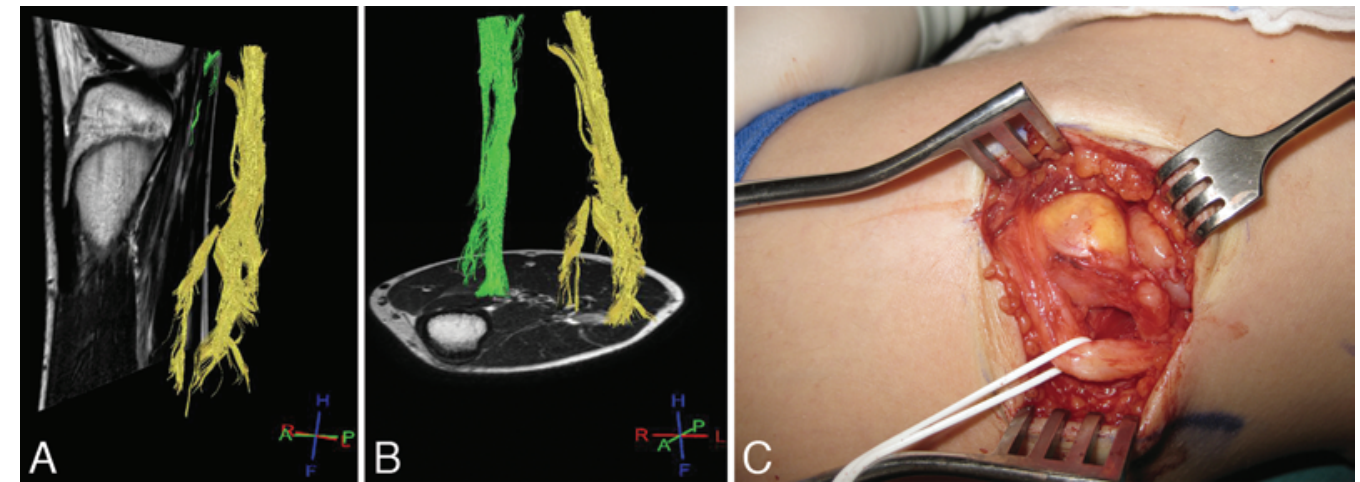

FIG. 3. Case 4. A and B: Three-dimensional DTT scans of a malignant PNST at the left peroneal nerve. The peroneal nerve (green) is split and the majority of the nerve is displaced laterally, whereas a smaller portion is running medially. The tibial nerve (yellow) is also visualized. C: Intraoperative photograph of the tumor also displayed in Figs. 3A and B. The tumor, the larger lateral fascicle group, and the second smaller fascicle group that passes the tumor mass medially are shown.
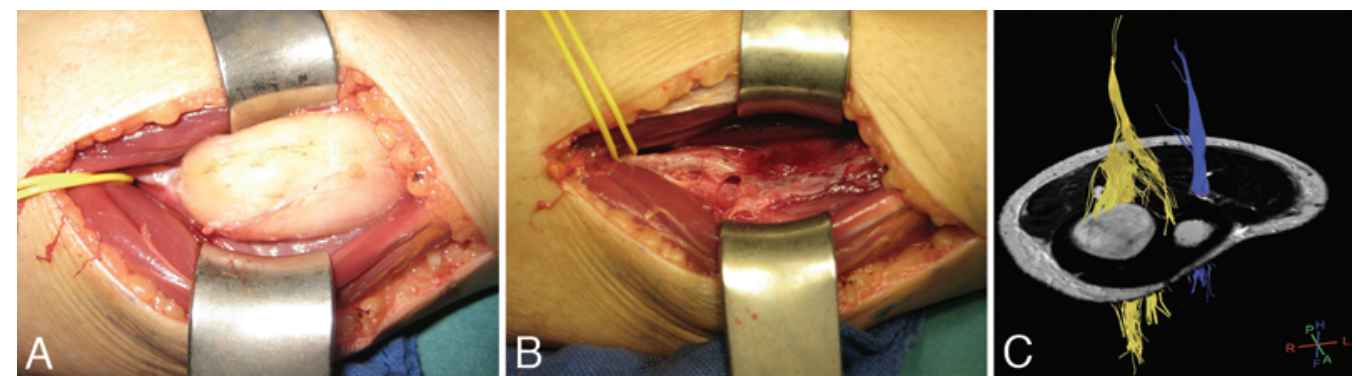

FIG. 4. Case 11. A: Photograph obtained in a patient with a schwannoma at the deep branch of the right peroneal nerve. The nerve is located deep to the tumor. B: Appearance of the peroneal nerve after tumor removal. The nerve was completely spread and flattened by the tumor. C: Preoperative DTT image of the right-lower leg. The peroneal nerve (yellow) appears flattened and broadened deep to the tumor. The tibial nerve (blue) is not affected.

of the nerve fascicles is complicated by severe stretching and thinning due to tumor growth. A further study is warranted to correlate the preoperative MR DTI study findings, intraoperative observations, and electrophysiological mapping of the tumor capsule.
Our experience demonstrates that DTT MRI scans in PNST patients facilitate the planning of effective tumor dissection and may reduce the risk of nerve injury during dissection by providing 3D imaging of the relationship between the nerve and tumor. The available 3D video se- 

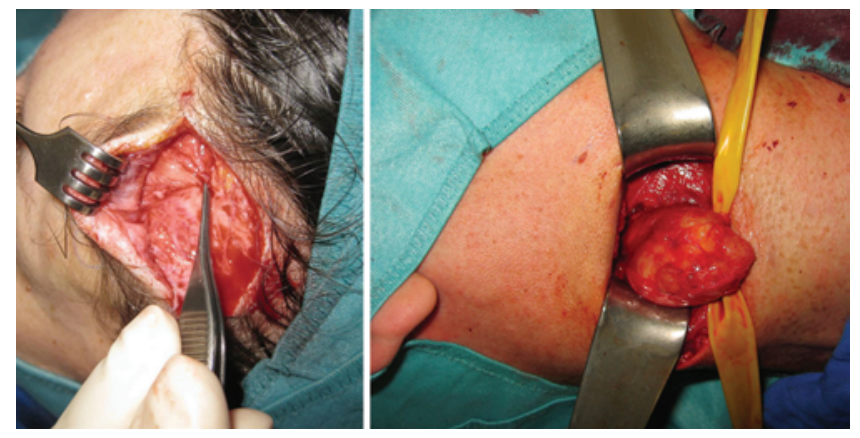

FIG. 5. Left: Case 3. Photograph obtained in a patient with small neurofibromata of the branches of the frontal nerve on the left side of the forehead. The forceps is pointing at one of the tumors, which were approached via a limited coronal incision in the hair-bearing area. No proper preoperative nerve visualization could be achieved in this patient due to the small size of the tumor and nerve branches. Right: Case 6. Photograph obtained in a patient with a focal malignant PNST of cranial nerve $X$ at the right side of the neck. No proper preoperative nerve visualization could be achieved in this patient due to proximity to the large cervical vessels.

quences were especially helpful for planning the surgical approach (Video 1).

Furthermore, preoperative evaluation of the nerve might provide insights for potential nerve reconstruction and guide the planning of nerve grafts. Possible further clinical applications of this technology for investigating peripheral nerve pathologies may include imaging nerve compression syndromes, e.g., the level of nerve compression in neuropathies of the ulnar nerve at the elbow. In patients with either traumatic or obstetric brachial plexus injuries, preoperative evaluation of the level of nerve disruption or injury, detection of fascicles in continuity, and their differentiation from ruptured nerve trunks could be performed. Another promising clinical application might be monitoring nerve regeneration, e.g., after nerve reconstruction or determining the timing of a functional muscle transplantation.

\section{Conclusions}

We were able to show that DTT properly visualizes peripheral nerve fascicles and their correct anatomical relation to PNSTs. DTT represents a valuable and promising new method for the preinterventional planning of nerve tumor resection. Further clinical applications will be explored. The limitations of DTT that we encountered include complex plexopathies, tumors in proximity to large vessels, extensive fiber splitting, and lesions smaller than $5 \mathrm{~mm}$.

\section{References}

1. Berman J: Diffusion MR tractography as a tool for surgical planning. Magn Reson Imaging Clin N Am 17:205-214, 2009

2. Cakir O, Arslan A, Inan N, Anık Y, Sarısoy T, Gumustas S, et al: Comparison of the diagnostic performances of diffusion parameters in diffusion weighted imaging and diffusion tensor imaging of breast lesions. Eur J Radiol 82:e801e806, 2013

3. Chhabra A, Thakkar RS, Andreisek G, Chalian M, Belzberg
AJ, Blakeley J, et al: Anatomic MR imaging and functional diffusion tensor imaging of peripheral nerve tumors and tumorlike conditions. AJNR Am J Neuroradiol 34:802-807, 2013

4. Demehri S, Belzberg A, Blakeley J, Fayad LM: Conventional and functional MR imaging of peripheral nerve sheath tumors: initial experience. AJNR Am J Neuroradiol 35:16151620,2014

5. Ducic I, Barrett DM, Al-Attar A: Benign peripheral nerve tumors: treatment algorithm and reconstructive options. Ann Plast Surg 63:156-161, 2009

6. Erturk SM, Ichikawa T, Kaya E, Yapici O, Ozel A, Mahmutoglu AS, et al: Diffusion tensor imaging of cysts, hemangiomas, and metastases of the liver. Acta Radiol 55:654-660, 2014

7. Furniss D, Swan MC, Morritt DG, Lim J, Khanna T, Way BL, et al: A 10-year review of benign and malignant peripheral nerve sheath tumors in a single center: clinical and radiographic features can help to differentiate benign from malignant lesions. Plast Reconstr Surg 121:529-533, 2008

8. Hems TE, Burge PD, Wilson DJ: The role of magnetic resonance imaging in the management of peripheral nerve tumours. J Hand Surg Br 22:57-60, 1997

9. Jambawalikar S, Baum J, Button T, Li H, Geronimo V, Gould ES: Diffusion tensor imaging of peripheral nerves. Skeletal Radiol 39:1073-1079, 2010

10. Kim DH, Murovic JA, Tiel RL, Kline DG: Operative outcomes of 546 Louisiana State University Health Sciences Center peripheral nerve tumors. Neurosurg Clin N Am 15:177-192, 2004

11. Kim DH, Murovic JA, Tiel RL, Moes G, Kline DG: A series of 397 peripheral neural sheath tumors: 30 -year experience at Louisiana State University Health Sciences Center. J Neurosurg 102: 246-255, 2005

12. Lehmann HC, Zhang J, Mori S, Sheikh KA: Diffusion tensor imaging to assess axonal regeneration in peripheral nerves. Exp Neurol 223:238-244, 2010

13. Li CS, Huang GS, Wu HD, Chen WT, Shih LS, Lii JM, et al: Differentiation of soft tissue benign and malignant peripheral nerve sheath tumors with magnetic resonance imaging. Clin Imaging 32:121-127, 2008

14. Nilsson J, Sandberg K, Søe Nielsen N, Dahlin LB: Magnetic resonance imaging of peripheral nerve tumours in the upper extremity. Scand J Plast Reconstr Surg Hand Surg 43:153-159, 2009

15. Nucifora PG, Verma R, Lee SK, Melhem ER: Diffusiontensor MR imaging and tractography: exploring brain microstructure and connectivity. Radiology 245:367-384, 2007

16. Russell SM: Preserve the nerve: microsurgical resection of peripheral nerve sheath tumors. Neurosurgery 61 (3 Suppl):113-118, 2007

17. Sandberg K, Nilsson J, Søe Nielsen N, Dahlin LB: Tumours of peripheral nerves in the upper extremity: a 22-year epidemiological study. Scand J Plast Reconstr Surg Hand Surg 43:43-49, 2009

18. Sheikh KA: Non-invasive imaging of nerve regeneration. Exp Neurol 223:72-76, 2010

19. Takagi T, Nakamura M, Yamada M, Hikishima K, Momoshima S, Fujiyoshi K, et al: Visualization of peripheral nerve degeneration and regeneration: monitoring with diffusion tensor tractography. Neuroimage 44:884-892, 2009

20. Thurnher MM, Law M: Diffusion-weighted imaging, diffusion-tensor imaging, and fiber tractography of the spinal cord. Magn Reson Imaging Clin N Am 17:225-244, 2009

21. Vargas MI, Viallon M, Nguyen D, Delavelle J, Becker M: Diffusion tensor imaging (DTI) and tractography of the brachial plexus: feasibility and initial experience in neoplastic conditions. Neuroradiology 52:237-245, 2010

22. Wiley J, Bhattacharyya AK, Guha A: Surgical management 
of peripheral nerve tumors, in Badie B (ed): Neurosurgical Operative Atlas: Neuro-Oncology, ed 2. New York: Thieme Medical Publishers, 2007, pp 76-84

23. Yamada K, Sakai K, Akazawa K, Yuen S, Nishimura T: MR tractography: a review of its clinical applications. Magn Reson Med Sci 8:165-174, 2009

\section{Disclosure}

The authors report no conflict of interest concerning the materials or methods used in this study or the findings specified in this paper.

\section{Author Contributions}

Conception and design: Aszmann, Schmidt. Acquisition of data: Schmidt, Kasprian, Amann. Analysis and interpretation of data: Aszmann, Schmidt. Drafting the article: Schmidt, Kasprian, Duscher. Critically revising the article: Aszmann, Schmidt, Duscher. Reviewed submitted version of manuscript: all authors. Approved the final version of the manuscript on behalf of all authors: Aszmann. Administrative/technical/material support: Kasprian. Study supervision: Aszmann.

\section{Supplemental Information \\ Videos}

Video 1, Media Player. http://mfile.akamai.com/21490/wmv/ digitalwbc.download.akamai.com/21492/wm.digitalsource-naregional/focus15-228_video_1.asx.

Video 1, Quicktime. http://mfile.akamai.com/21488/mov/ digitalwbc.download.akamai.com/21492/qt.digitalsource-global/ focus15-228_video_1.mov.

\section{Previous Presentations}

This work was presented in part at the 2nd European Plastic Surgery Research Council, Hamburg, Germany, August 26-29, 2010, and the 6th Congress of the World Society for Reconstructive Microsurgery, Helsinki, Finland, June 29-July 2, 2011.

\section{Correspondence}

Oskar C. Aszmann, Division of Plastic and Reconstructive Surgery, Medical University of Vienna, Waehringer Guertel 18-20, A-1090 Vienna, Austria. email: oskar.aszmann@meduniwien. ac.at. 